\title{
ON THE RESIDUAL NILPOTENCE OF SOME VARIETAL PRODUCTS
}

\author{
BY \\ GILBERT BAUMSLAG(1)
}

1. Introduction. A nonempty class $\mathfrak{v}$ of groups is a variety if it is closed with respect to the formation of subgroups, factor groups and cartesian products. If $G$ is any group, we define $\mathfrak{v}(G)$ to be the intersection of all normal subgroups $N$ of $G$ such that $G / N \in \mathfrak{p}$; it is not difficult to show that $G / \mathfrak{p}(G) \in \mathfrak{p}$.

Now suppose $\left\langle A_{\lambda} ; \lambda \in \Lambda\right\rangle$ is a given family of groups. Let $P$ be the free product of the groups $A_{\lambda}$; then

$$
F=P / \mathfrak{v}(P)
$$

is called the free $\mathfrak{v}$-product of the groups $G_{\lambda}$ (cf. S. Moran [1]). If the groups $A_{\lambda}$ are all infinite cyclic, then $F$ is termed a free $\mathfrak{v}$-group and the cardinality $|\Lambda|$ of $\Lambda$ is the rank of $F$. The purpose of this paper is to show that, for certain varieties $\mathfrak{v}$, the residual nilpotence $\left({ }^{2}\right)$ of a free $\mathfrak{v}$-group of infinite rank implies the residual nilpotence of the free $\mathfrak{b}$-product of every family of torsion-free abelian groups.

We need the notion of the composition $\mathfrak{u} \mathfrak{w}$ of two varieties $\mathfrak{u}$ and $\mathfrak{w}$ introduced by Hanna Neumann [2]. By definition uw consists of those groups $G$ which possess a normal subgroup $N \in \mathfrak{u}$ such that $G / N \in \mathfrak{w}$; notice that $\mathfrak{u} \mathfrak{w}$ is itself a variety (Hanna Neumann [2]).

Now let $\mathfrak{a}$ be the variety of all abelian groups and let $\mathfrak{u}$ be any given variety. The purpose of this paper is the proof of the

THEOREM. The free $\mathfrak{u} \mathfrak{a}$-product $F$ of every family $\left\langle A_{\lambda} ; \lambda \in \Lambda\right\rangle$ of torsion-free abelian groups is residually torsion-free nilpotent if and only if some free $\mathfrak{u} a$-group $X$ of countably infinite rank is residually torsion-free nilpotent $\left({ }^{3}\right)$.

K. W. Gruenberg [3] has shown that every free $\mathfrak{a}^{n}$-group is residually torsionfree nilpotent $(n=1,2, \cdots)$, where inductively

$$
\mathfrak{a}^{r+1}=\mathfrak{a} \mathfrak{a}^{r} \quad(r>0) .
$$

Consequently, by the theorem, the free $a^{n}$-product of any family of torsion-free

Received by the editors November 9, 1962.

(1) Sponsored by the National Science Foundation, Grant GP 27.

(2) If $\mathscr{P}$ is a property pertaining to groups then, according to P. Hall [9], a group $G$ is residually $\mathscr{P}$ if every element $x \in G(x \neq 1)$ can be omitted from a normal subgroup $N_{x}$ such that $G / N_{x}$ is $\mathscr{P}$.

(3) Cf. Theorem 6.2 of K. W. Gruenberg [3]. 
abelian groups is residually torsion-free nilpotent $(n=1,2, \cdots)$. When $n=2$ this reduces to a theorem of Rimhak Ree [4]; the more general result answers the question raised by Ree in [4, p. 394].

It may be well to mention that we first prove the theorem when the $A_{\lambda}$ are free abelian (Proposition 1) and then make use of an embedding theorem of A. I. Mal'cev [5] to prove the theorem in general (see Proposition 2).

2. The proof of Proposition 1. This is the first stage of the proof of the theorem of this paper. Incidentally, Proposition 1 seems interesting in itself.

Proposition 1. The free $\mathfrak{u} a$-product $F$ of a family $\left\langle A_{\lambda} ; \lambda \in \Lambda\right\rangle$ of free abelian groups is residually a free $\mathfrak{u a}$-group.

The proof of Proposition 1 will be accomplished by introducing four lemmas. We begin with the first of these, which amounts to a generalization of a theorem of Gilbert Baumslag [6].

LEMMA 1. Let $F$ be the free $\mathfrak{u} a$-product of a family $\left\langle A_{\lambda} ; \lambda \in \Lambda\right\rangle$ of free abelian groups. Furthermore, let $U_{\lambda}$ be an infinite cyclic subgroup of $A_{\lambda}$ for each $\lambda \in \Lambda$. Then $E$, the subgroup generated by the $U_{\lambda}$, is a free $\mathfrak{u} a-g r o u p ;$ indeed $E$ is the free $\mathfrak{u}$ a-product of its subgroups $U_{\lambda}$.

Proof. Suppose

Let

$$
U_{\lambda}=\operatorname{gp}\left(u_{\lambda}\right) \quad(\lambda \in \Lambda)
$$

$$
W_{\lambda}=\operatorname{gp}\left(w_{\lambda}\right) \quad(\lambda \in \Lambda)
$$

be infinite cyclic groups and let $W$ the free $\mathfrak{u} a$-product of the $W_{\lambda}(\lambda \in \Lambda)$.

Now for every group $C \in \mathfrak{u} \mathfrak{a}$ and every system $\phi_{\lambda}$ of homomorphisms of $A_{\lambda}$ into $C(\lambda \in \Lambda)$ there is a homomorphism $\phi$ of $F$ into $C$ which coincides with $\phi_{\lambda}$ on $A_{\lambda}$ (cf., e.g., S. Moran [1]). Since the $A_{\lambda}$ are free abelian it is easy to concoct a homomorphism $\phi_{\lambda}$ of $A_{\lambda}$ to $W_{\lambda}$ such that $u_{\lambda} \phi_{\lambda} \neq 1$, say

$$
u_{\lambda} \phi_{\lambda}=w_{\lambda}^{r_{\lambda}} \quad\left(r_{\lambda} \neq 0\right) \text {. }
$$

Let $\phi$ be the homomorphism of $F$ into $W$ continuing the $\phi_{\lambda}$. We claim, that the restriction of $\phi$ to $E$ is a monomorphism.

To see this, notice that

$$
E \phi=\operatorname{gp}\left(w_{\lambda}^{r \lambda} ; \lambda \in \Lambda\right) .
$$

By Theorem 3 of Gilbert Baumslag [6], $E \phi$ is the free $\mathfrak{u}$ a-product of the groups $\operatorname{gp}\left(w_{\lambda}^{r \lambda}\right)(\lambda \in \Lambda)$. Hence the mappings

$$
\theta_{\lambda}: w_{\lambda}^{r \lambda} \rightarrow u_{\lambda}
$$

can be extended to an epimorphism $\theta$ of $E \phi$ to $E$. Since $\phi \theta$ is the trivial auto- 
morphism when restricted to $E$, the restriction of $\phi$ to $E$ is a monomorphism; this completes the proof.

It is worthwhile, at this point, to explain the motivation of the introduction of Lemma 1. We shall prove Proposition 1 by showing that if $f \in F(f \neq 1)$, then there exists an endomorphism $\hat{\eta}$ of $F$ such that $F \hat{\eta}$ is a free $\mathfrak{u} \mathfrak{a}$-group and $f \hat{\eta} \neq 1$; it is Lemma 1 that affords us with subgroups of $F$ which are free $\mathfrak{u} a$-groups. The remaining lemmas that we shall need before proceeding to the actual proof of Proposition 1 are aimed at establishing the existence of such an endomorphism $\hat{\eta}$.

Lemma 2 (K. W.Gruenberg [3]). Let $\Lambda$ be a totally ordered set and let $P$ be the free product of a family $\left\langle A_{\lambda} ; \lambda \in \Lambda\right\rangle$ of torsion-free abelian groups. Then $P^{\prime}$, the commutator subgroup of $P$, is freely generated by

$$
\begin{aligned}
S=\left\{\left[b_{\lambda}, b_{\mu}, \cdots, b_{\rho}\right] \mid b_{\lambda} \in A_{\lambda}, b_{\mu} \in A_{\mu}, \cdots, b_{\rho} \in A_{\rho},\right. \\
\\
\left.\qquad b_{\lambda} \neq 1, b_{\mu} \neq 1, \cdots, b_{\rho} \neq 1, \lambda>\mu, \mu<\cdots<\rho\right\} .
\end{aligned}
$$

[We have used in Lemma 2 the usual commutator notation. Consequently,

$$
[x, y]=x^{-1} y^{-1} x y
$$

and, inductively,

$$
\left.\left[x_{1}, x_{2}, \cdots, x_{n}\right]=\left[\left[x_{1}, x_{2}, \cdots, x_{n-1}\right], x_{n}\right] \quad(n>2) \cdot\right]
$$

We need next a simple combinatorial fact concerning certain sequences of integers.

LEMMA 3. Let

$$
\left(e_{i 1}, e_{i 2}, \cdots, e_{i r}\right) \quad(i=1,2, \cdots, k)
$$

be distinct r-termed sequences of integers. Then there exist integers

$$
c_{1}, c_{2}, \cdots, c_{r}
$$

such that the sums

$$
\sum_{j=1}^{r} e_{i j} c_{j} \quad(i \in\{1,2, \cdots, k\})
$$

are also distinct.

Proof. The proof of Lemma 3 is a straightforward argument by induction on $r$. To begin with, if $r=1$, then we need only put

$$
c_{1}=c_{2}=\cdots=c_{r}=1
$$

to obtain the desired result.

Thus let us suppose $r>1$ and inductively choose 
so that

$$
c_{1}, c_{2}, \cdots, c_{r-1}
$$

$$
\sum_{j=1}^{r-1} e_{h j} c_{j}=\sum_{j=1}^{r-1} e_{i j} c_{j}
$$

if and only if

$$
\left(e_{h 1}, e_{h 2}, \cdots, e_{h r-1}\right)=\left(e_{i 1}, e_{i 2}, \cdots, e_{i r-1}\right),
$$

where $h, i \in\{1,2, \cdots, k\}$. We are left now with the choice of $c_{r}$. To this end let

$$
h, i \in\{1,2, \cdots, k\} \quad(h \neq i) .
$$

Consider the equation

$$
\sum_{j=1}^{r-1} e_{h j} c_{j}+x e_{h r}=\sum_{j=1}^{r-1} e_{i j} c_{j}+x e_{i r}
$$

This equation (1) has at most one solution. To see this notice that the sequences

$$
\left(e_{h 1}, e_{h 2}, \cdots, e_{h r}\right), \quad\left(e_{i 1}, e_{i 2}, \cdots, e_{i r}\right)
$$

are distinct. So if $e_{h r}=e_{i r}$,

$$
\sum_{j=1}^{r-1} e_{h j} c_{j} \neq \sum_{j=1}^{r-1} e_{i j} c_{j}
$$

and (1) does not have a solution. On the other hand, if $e_{h r} \neq e_{i r}$, then (1) clearly has a single solution. It follows that there are at most finitely many integers which satisfy at least one of the finitely many equations

$$
\sum_{j=1}^{r-1} e_{h j} c_{j}+x e_{h r}=\sum_{j=1}^{r-1} e_{i j} c_{j}+x e_{i r} \quad(h, i \in\{1,2, \cdots, k\}, h \neq i) .
$$

We can therefore choose $c_{r}$ in accordance with the requirements of the lemma and this then completes the proof.

Suppose now that we assume the notation of Lemma 2. We shall say that

$$
s\left(=\left[b_{\lambda}, b_{\mu}, \cdots, b_{\rho}\right]\right) \in S
$$

involves $A_{\alpha}(\alpha \in \Lambda)$ if

$$
\alpha \in\{\lambda, \mu, \cdots, \rho\},
$$

and we say that the $A_{\alpha}$ contribution to $s$ is $b_{\alpha}$. Then the following lemma holds.

LEMMA 4. Let $\alpha$ be a fixed element of $\Lambda$ and let $b$ be part of $a$ basis for $A_{\alpha}$. Further let

$$
s_{1}, s_{2}, \cdots, s_{k} \quad\left(s_{i} \neq s_{j} \text { if } i \neq j\right)
$$


be elements of $S$ all of which involve $A_{\alpha}$. Then there exists an endomoromorphism $\eta_{\alpha}$ of $P$, which is the identity on $A_{\beta}(\beta \neq \alpha)$, such that

(i) $s_{1} \eta_{\alpha}, s_{2} \eta_{\alpha}, \cdots, s_{k} \eta_{\alpha}$ are distinct elements of $S$,

(ii) $s_{1} \eta_{\alpha}, s_{2} \eta_{\alpha}, \cdots, s_{k} \eta_{\alpha}$ involve $A_{\alpha}$, and

(iii) $A_{\alpha} \eta_{\alpha} \leqq \mathrm{gp}(b)$.

Proof. Let $a_{i}$ be the $A_{\alpha}$-contribution of $s_{i}(i=1,2, \cdots, k)$. Then we choose a subset

$$
b_{1}=b, b_{2}, \cdots, b_{r}
$$

of a basis $B$ of $A_{\alpha}$ such that

$$
a_{i} \in \operatorname{gp}\left(b_{1}, b_{2}, \cdots, b_{r}\right) \quad(i=1,2, \cdots, k) .
$$

Then

$$
a_{i}=b_{1}^{e_{i 1}} b_{2}^{e_{i 2}} \cdots b_{r}^{e_{i}^{r}} \quad(i=1,2, \cdots, k) .
$$

Consider the $k$ sequences

$$
\left(e_{i 1}, e_{i 2}, \cdots, e_{i r}\right) \quad(i=1,2, \cdots, k) .
$$

Since $a_{i} \neq 1(i=1,2, \cdots, k)$, none of the sequences $\left(e_{i 1}, e_{i 2}, \cdots, e_{i r}\right)$ consists entirely of zeros. If we add to the sequences (3) the sequence

$$
(0,0, \cdots, 0) \text {, }
$$

then it follows from Lemma 3 that we can find integers $c_{1}, c_{2}, \cdots, c_{r}$ such that, firstly,

$$
\sum_{j \infty 1}^{r} e_{i j} c_{j} \neq 0
$$

and secondly, if $h, i \in\{1,2, \cdots, k\}$,

(5) $\sum_{j \infty 1}^{r} e_{h j} c_{j}=\sum_{j \infty 1}^{r} e_{i j} c_{j}$ if and only if $\left(e_{h 1}, e_{h 2}, \cdots, e_{h r}\right)=\left(e_{i 1}, e_{i 2}, \cdots, e_{i r}\right)$.

We are now in a position to define $\eta_{\alpha}$. To begin with we define the effect of $\eta_{\alpha}$ on $A_{\beta}(\beta \neq \alpha)$ to be the identity mapping. Next we define the action of $\eta_{\alpha}$ on $A_{\alpha}$ by specifying its action on a basis of $A_{\alpha}$. We consider the basis $B$ involved in (2); thus we put

$$
x \eta_{\alpha}=1 \text { if } x \notin\left\{b_{1}, b_{2}, \cdots, b_{v}\right\} \quad(x \in B),
$$

and, finally, define

$$
b_{i} \eta_{\alpha}=b^{c_{i}} \quad(i=1,2, \cdots, r) .
$$

By (4), (5) and Lemma 2 it follows that if $h, i \in\{1,2, \cdots, r\}$, then 


$$
a_{h} \eta_{\alpha}=a_{i} \eta_{\alpha} \text { if and only if } a_{h}=a_{i} .
$$

This completes the proof of Lemma 4 (cf. Lemma 2).

It is not difficult now to deduce Proposition 1. Thus let us suppose that $F$ is the free $\mathfrak{u}$ a-product of the free abelian groups $A_{\lambda}(\lambda \in \Lambda)$. Then

$$
F=P / \mathfrak{u} \mathfrak{a}(P),
$$

where $P$ is the free product of the groups $A_{\lambda}(\lambda \in \Lambda)$. It is therefore sufficient, for the proof of Proposition 1, to show that if

$$
w \in P, w \notin \mathfrak{u} a(P)
$$

then there exists a homomorphism $\eta$ of $P$ into a free $\mathfrak{u}$ a-group such that

$$
w \eta \neq 1,
$$

since the kernel $K$ of $\eta$ will necessarily contain $\mathfrak{u} \mathfrak{a}(P)$.

If $w \notin P^{\prime}$, then it is easy, on noting that $P / P^{\prime}$ is free abelian, to find a homomorphism $\eta$ of $P$ into an infinite cyclic group (i.e., a free $\mathfrak{u}$ a-group) so that $w \eta \neq 1$.

Thus we may suppose $w \in P^{\prime}$. Now, by Lemma 2,

$$
w=s_{1}^{\varepsilon_{1}} s_{2}^{\varepsilon_{2}} \cdots s_{k}^{\varepsilon_{k}} \quad\left(\varepsilon_{i}= \pm 1, s_{i} \in S\right) .
$$

It follows easily, by a repeated application of Lemma 4, that there exists an endomorphism $\eta^{*}$ of $P$ such that

(i) $A_{\lambda} \eta^{*}$ is an infinite cyclic subgroup of $A_{\lambda}(\lambda \in \Lambda)$,

(ii) $s_{i} \eta^{*} \in S(i=1,2, \cdots, k)$,

(iii) $s_{i} \eta^{*}=s_{j} \eta^{*}$ if and only if $s_{i}=s_{j}(i, j \in\{1,2, \cdots, k\})$.

Now by Lemma $2, S$ is a free set of generators of the free group

$$
P^{\prime}=\mathfrak{a}(P) \text {. }
$$

Consequently, as $\eta^{*}$ is one-to-one on

$$
\left\{s_{1}, s_{2}, \cdots, s_{k}\right\},
$$

by (iii), there is certainly an automorphism $\mu$ of $\mathfrak{a}(P)$ such that

$$
s_{i} \mu=s_{i} \eta^{*} \quad(i=1,2, \cdots, k) .
$$

Therefore

$$
w \eta^{*}(=w \mu) \notin \mathfrak{u}(\mathfrak{a}(P))(=\mathfrak{u} \mathfrak{a}(P))
$$

since

$$
w \notin \mathfrak{u} \mathfrak{a}(P) .
$$

Since $\mathfrak{u} \mathfrak{a}(P)$ is fully invariant the mapping

$$
\eta: x \rightarrow x \eta * \mathfrak{u a}(P)
$$


is a homomorphism of $P$ into $P \eta^{*} \mathfrak{u} \mathfrak{a}(P) / \mathfrak{u a}(P)$. But by (i) and Lemma $1, P \eta$ is a free $\mathfrak{u} a$-group. Furthermore, by (6),

$$
w \eta \neq 1 .
$$

This completes the proof of Proposition 1.

We would like to place on record the obvious conjecture that arises in connection with Proposition 1.

Conjecture. Let $G$ be the free $\mathfrak{u}$-product of a family of free $(\mathfrak{u} \cap \mathfrak{a})$-groups. Then $G$ is residually a free $\mathfrak{u}$-group.

3. Proposition 2. A group $K$ is termed radical if extraction of roots is always possible in $K$. A. I. Mal'cev [5] has shown that a torsion-free nilpotent group can always be embedded in a torsion-free nilpotent radical group of the same class. Suppose now that $K$ is a torsion-free nilpotent group and that $K^{*}$ is a torsionfree nilpotent radical group containing $K ; K^{*}$ is called a completion of $K$ if every radical subgroup of $K^{*}$ containing $K$ coincides with $K^{*}$. It is easy to see then that every torsion-free nilpotent group $K$ has a completion $K^{*}$; moreover two completions of $K$ are isomorphic (A. I. Mal'cev [5]). We need some additional information about $K^{*}$.

Proposition 2. Let $K$ be a torsion-free nilpotent group. If $K$ belongs to a variety $\mathfrak{v}$ then so does every completion $K^{*}$ of $K$.

Proof. It is easy to see that if every finitely generated subgroup of $K^{*}$ lies in $\mathfrak{v}$ then so does $K^{*}$ (cf. e.g. Hanna Neumann [2]).

Thus let $H$ be a finitely generated subgroup of $K^{*}$ :

Put

$$
H=\operatorname{gp}\left(a_{1}, a_{2}, \cdots, a_{n}\right)
$$

$$
L=H \cap K
$$

By a theorem of A. I. Mal'cev (cf. e.g. A. G. Kurosh [7, p. 248, Volume 2]), there is an integer $r$ such that $a_{i}^{r} \in K(i=1,2, \cdots, n)$, i.e.,

$$
a_{i}^{r} \in L \text {. }
$$

Let $p$ be a prime chosen so that

$$
(p, r)=1 \text {. }
$$

Now, by a theorem of K. W. Gruenberg [3] the normal subgroups of p-power index in a finitely generated torsion-free nilpotent group intersect in the identity. Thus if $x \in H(x \neq 1)$ we can find $N$, normal in $H$, such that $x \notin N$ and $H / N$ is of order a power of $p$. But by (7) and (8) it follows that

$$
a_{i} \in L N \quad(i=1,2, \cdots, n) .
$$


Hence

But

$$
L N / N=H / N
$$

$$
L N / N \cong L / L \cap N .
$$

Therefore $\mathfrak{v}(H) \leqq N$ since $L$ (and so also $L / L \cap N) \in \mathfrak{p}$. Consequently

$$
x \notin \mathfrak{v}(H),
$$

and so $\mathfrak{v}(H)=1$, i.e., $H \in \mathfrak{v}$. This completes the proof of Proposition 2 .

4. The proof of the main result. We recall that the object of this paper is the proof of the

THEOREM. Let $\mathfrak{u}$ be any variety and let $\mathfrak{a}$ be the variety of abelian groups. Then the free $\mathfrak{u} \mathfrak{a}$-product of every family of torsion-free abelian groups is residually torsion-free nilpotent if and only if some free $\mathfrak{u a}$-group of countably infinite rank is residually torsion-free nilpotent.

Proof. The one part of the theorem is trivial.

For the other let us suppose that some free $\mathfrak{u} \mathfrak{a}$-group of infinite rank is residually torsion-free nilpotent. Then, clearly, every free $\mathfrak{u} \mathfrak{a}$-group is residually torsion-free nilpotent.

Now let $\left\langle A_{\lambda} ; \lambda \in \Lambda\right\rangle$ be a family of torsion-free abelian groups and let $F$ be their free $\mathfrak{u} \mathfrak{a}$-product. Furthermore, let

$$
f \in F \quad(f \neq 1) .
$$

We can find (cf., e.g., L. Fuchs [8]) free abelian subgroups $B_{\lambda}$ of $A_{\lambda}$ such that

(i) $A_{\lambda} / B_{\lambda}$ is periodic $(\lambda \in \Lambda)$,

(ii) $f \in \operatorname{gp}\left(B_{\lambda} ; \lambda \in \Lambda\right)$.

We choose, for each $\lambda \in \Lambda$, an isomorphic copy $\bar{B}_{\lambda}$ of $B_{\lambda}$ and consider their free $\mathfrak{u} \mathfrak{a}$-product $\bar{B}$. Then, by Proposition $1, F$ is residually torsion-free and nilpotent. Consequently, $\bar{B}$ is a subgroup of a cartesian product $C$ of torsion-free nilpotent groups $T_{i}(i \in I)$ (cf., e.g., K. W. Gruenberg [3]):

$$
C=\prod_{i=1}^{\infty} T_{i} .
$$

Let $T_{i}^{*}$ be the completion of $T_{i}$ and let $C^{*}$ be the cartesian product of the $T_{i}^{*}$ :

$$
C^{*}=\prod_{i=1}^{\infty} T_{i}^{*}
$$

Now it is easy to see, on noting that $C^{*}$ is radical and torsion-free, that there is a completion $C_{\lambda}$ of $\bar{B}_{\lambda}$ in $C^{*}$. So by the choice of the subgroup $B_{\lambda}$ of $A_{\lambda}$ (see (i) 
above) it follows that there is a monomorphism $\sigma_{\lambda}$ of $A_{\lambda}$ into $C_{\lambda}$ mapping $B_{\lambda}$ isomorphically onto $\bar{B}_{\lambda}$; let $\tilde{\sigma}_{\lambda}$ be the restriction of $\sigma_{\lambda}$ to $B_{\lambda}$.

The groups $T_{i} \in \mathfrak{u} \mathfrak{a}$; hence by Proposition $2, T_{i}^{*} \in \mathfrak{u} \mathfrak{a}$. But then $C^{*} \in \mathfrak{u} \mathfrak{a}$. This means that the system of mappings $\sigma_{\lambda}(\lambda \in \Lambda)$ can be continued to a homomorphism $\sigma$ of $F$ into $C^{*}$.

Let $B$ be the subgroup generated by the $B_{\lambda}(\lambda \in \Lambda)$. Then $\sigma$ induces an epimorphism $\tilde{\sigma}$ from $B$ to $\bar{B}$. Indeed we claim that $\tilde{\sigma}$ is an isomorphism. To see this we recall that $\bar{B}$ is the free $\mathfrak{u} \mathfrak{a}$-product of the groups $\bar{B}_{\lambda}$. Hence the homomorphisms

$$
\tilde{\sigma}_{\lambda}^{-1}: \bar{B}_{\lambda} \rightarrow B_{\lambda}
$$

can be extended to a homomorphism $\bar{\sigma}$ from $\bar{B}$ to $B$. It follows immediately that $\tilde{\sigma}$ and $\bar{\sigma}$ are mutually inverse; hence $\tilde{\sigma}$ is one-to-one (and so $B$ is the free $\mathfrak{u} \mathfrak{a}$-product of its subgroups $B_{\lambda}(\lambda \in \Lambda)$; this represents a partial generalization of a theorem of Gilbert Baumslag [6]).

But now the one-to-oneness of $\tilde{\sigma}$ implies

$$
f \sigma(=f \tilde{\sigma}) \neq 1 \text {. }
$$

So there is a normal subgroup $N$ of $F$, which does not contain $f$, such that $F / N$ is torsion-free nilpotent. This completes the proof of the theorem.

\section{REFERENCES}

1. S. Moran, Associative operations on groups. I, Proc. London Math. Soc. 6 (1956), 581-596.

2. Hanna Neumann, On varieties of groups and their associated near-rings, Math. Z. 65 (1956), 36-69.

3. K. W. Gruenberg, Residual properties of infinite soluble groups, Proc. London Math. Soc. 7 (1957), 29-62.

4. R. Ree, Commutator groups of free products of torsion-free abelian groups, Ann. of Math. (2) 66 (1957), 380-394.

5. A. I. Mal'cev, Nilpotent torsion-free groups, Izv. Akad. Nauk SSSR. 13 (1949), 201-212.

6. G. Baumslag, Some subgroup theorems for free $\mathfrak{v}$-groups, Trans. Amer. Math. Soc. 108 (1963), 516-525.

7. A. G. Kurosh, The theory of groups, Chelsea, New York, 1955. (English transl.)

8. L. Fuchs, Abelian groups, Pergammon, Budapest, 1958.

9. P. Hall, The splitting properties of relatively free groups, Proc. London Math. Soc. 4 (1954), 343-356.

NEW YoRK UNIVERSITY, NeW YoRK, New YoRK 